\title{
VALIDAÇÃO DO MÉTODO DE QUANTIFICAÇÃO DO ÓLEO DE CAFÉ TORRADO NANOENCAPSULADO EM POLI(L- ÁCIDO LÁTICO)
}

\author{
S. J. de SOUZA ${ }^{1}$, K. C. KAUFMANN ${ }^{1}$, M. F. de SOUZA ${ }^{1}$, E. B. FERIBERGER ${ }^{3}$, P. H. H. de \\ ARAÚJO2 ${ }^{2}$, O. H. GONÇALVES ${ }^{3}$, F. V. LEIMANN ${ }^{3 *}$ \\ ${ }^{1}$ Universidade Tecnológica Federal do Paraná-Campus Campo Mourão, Departamento \\ Acadêmico de Alimentos; \\ 2 Universidade Federal de Santa Catarina, Departamento de Engenharia Química \\ ${ }^{3}$ Universidade Tecnológica Federal do Paraná-Campus Campo Mourão, Programa de Pós- \\ Graduação em Tecnologia de Alimentos. \\ E-mail para contato: fernandaleimann@utfpr.edu.br
}

\begin{abstract}
RESUMO - A nanoencapsulação é uma técnica promissora para garantir a proteção de compostos voláteis e misturas de lipídeos naturais como óleo de café torrado. Neste trabalho nanocápsulas de poli(L-ácido lático) (PLLA) contendo óleo de café torrado foram obtidas pela técnica de miniemulsificação/evaporação do solvente. Óleo presente na formulação foi quantificado em termos de rendimento de recuperação por Espectrofotometria UV-Vis. O método foi validado pelas seguintes figuras de mérito: linearidade, limite de detecção, limite de quantificação, precisão, exatidão e especificidade. Os resultados obtidos permitiram concluir que a recuperação do óleo total encapsulado nas nanocápsulas de PLLA pode ser determinada na faixa de 0,500 a $0,100 \mathrm{mg} / \mathrm{mL}$.
\end{abstract}

\section{INTRODUÇÃO}

A fração oleosa de café torrado é composta principalmente por ácidos graxos livres e esterificados, diterpenos, esteróis e compostos voláteis. As propriedades funcionais do café e de seus subprodutos foram extensivamente avaliadas já que estes são amplamente utilizados pela indústria de alimentos (Clarke, 2001). Além disso, tem havido interesse na utilização do óleo de café torrado para melhorar as propriedades sensoriais de preparações de café solúvel já que o aroma é o principal fator na decisão de compra pelos consumidores (Bhumiratana et al., 2011). O aroma do café solúvel (instantâneo) difere do café torrado e moído devido às condições severas utilizadas durante os processos de extração e de secagem (Farah, 2009). Madene et al. (2006) destacam que a incorporação de pequenas quantidades de flavors em alimentos pode influenciar de forma efetiva o produto acabado e sua qualidade, reforçando a necessidade do fornecimento de produtos altamente aromáticos. $\mathrm{O}$ desenvolvimento de metodologias de nanoencapsulação tem aberto possibilidades promissoras para a indústria alimentícia, destacando-se a miniemulsificação/evaporação do solvente. Diferentes estudos mostram que a técnica de miniemulsificação é efetiva para encapsular compostos lipídicos ou lipossolúveis (da Silva-Buzanello et al., 2015). O poli(L-ácido lático) (PLLA) é um polímero biocompatível e biodegradável que durante a degradação in vivo tem sua cadeia polimérica quebrada em unidades monoméricas de ácido láctico que é convertido em glicose pelo fígado, 
que por sua vez é usada como fonte de energia para o corpo. O uso de nanopartículas de PLLA é, portanto, seguro e desprovida de qualquer toxicidade grave (Mahapatro e Singh, 2011). O PLLA tem sido largamente aplicado em embalagens de alimentos(Tawakkal et al., 2014) e para encapsular diferentes compostos para aplicação em alimentos como a vitamina $\mathrm{E}$ (Chaiyasat et al., 2013). Durante o processo de encapsulação os compostos aromáticos podem ser degradados ou perdidos sendo necessário realizar a quantificação do óleo efetivamente presente na formulação (da Silva-Buzanello et al, 2015). De acordo com Chorny et al. (2002) o rendimento de recuperação do composto encapsulado pode ser definido com a relação entre a quantidade do composto inicialmente adicionada à formulação e a quantidade real determinada após o procedimento de encapsulação. A validação de um procedimento analítico pode ser atestada através da determinação de parâmetros conhecidos como figuras de mérito. Esses parâmetros, dependendo de onde o método será aplicado, do seu propósito e ou do órgão de físcalização a que estará sujeito podem variar, sendo as principais: exatidão, precisão, sensibilidade, seletividade (ou especificidade), linearidade e limites de detecção e de quantificação (Valderrama et al., 2009). Somente a partir da análise destas figuras de mérito é possível garantir que o método proposto é válido para quantificar o composto de interesse nas condições de encapsulação a que foi submetido. Desta forma o objetivo do presente trabalho é a validação da metodologia de rendimento de recuperação do óleo de café torrado nanoencapsulado em poli(L-ácido lático) (PLLA).

\section{MATERIAL E MÉTODOS}

\subsection{Materiais}

L-lactídeo (Purac), octanoato de estanho (Sigma-Aldrich): síntese do PLLA. Diclorometano (Vetec, 99,5\% de pureza), metanol (Pró-Quimios, 99,5\% de pureza), lecitina de soja (Alfa Aesar) para produção e análise das nanocápsulas. O óleo de café torrado foi gentilmente cedido pela Café Iguaçu Ltda, de Cornélio Procópio, Paraná.

\subsection{Produção e caracterização das nanocápsulas}

O poli(L-ácido lático) (PLLA) foi sintetizado pelo método de polimerização por abertura de anel de L-lactídeo utilizando octanoato de estanho como catalisador, de acordo com da Silva-Buzanello et al. (2015). Este foi utilizado como polímero encapsulante. A técnica de miniemulsificação/evaporação do solvente foi utilizada de acordo com a descrição de Leimann et al. (2013). O óleo de café torrado (131 mg) foi encapsulado em PLLA (262 $\mathrm{mg})$, lecitina $(89,6 \mathrm{mg})$ foi utilizada como surfactante, diclorometano $(11,56 \mathrm{~g})$ como solvente orgânico e água destilada $(22,44 \mathrm{~g})$ como meio contínuo. Nanopartículas sem óleo foram preparadas para servirem como branco na análise de UV-Vis. A miniemulsificação foi realizada nas seguintes condições: sonicação de $180 \mathrm{~s}$ e amplitude de $100 \%$ (30 s sonicação, $10 \mathrm{~s}$ pausa) (Fisher-Scientific - Ultrasonic Dismembrator $120 \mathrm{~W}$ com ponta de $1 / 8$ '). Após a preparação a miniemulsão o solvente foi evaporado $\left(18 \mathrm{~h} \mathrm{a} 40{ }^{\circ} \mathrm{C}\right.$ em shaker). $\mathrm{O}$ diâmetro médio em intensidade (Dz) e índice de polidispersão (IPD) das nanocápsulas foram determinados por Espalhamento Dinâmico de Luz (Malvern - Nanosizer, Nano Series) (Malvern Instruments, 2011). A determinação do rendimento de recuperação do óleo nanoencapsulado foi realizada por Espectrofotometria de Ultravioleta-Visível (UV-Vis, PG Instruments e Hitachi U-1900). Uma alíquota de $1 \mathrm{~mL}$ de dispersão de nanocápsulas foi seca 
em estufa $\left(40{ }^{\circ} \mathrm{C}, 2 \mathrm{~h}\right)$. Adicionou-se $1 \mathrm{~mL}$ de diclorometano e $1 \mathrm{~mL}$ de metanol. Filtrou-se a solução com filtros de seringa de nylon $(0,45 \mu \mathrm{m}$, Maxcrom) e então diluiu-se 10 vezes e a amostra foi analisada no UV-Vis. O mesmo procedimento foi utilizado para as nanopartículas sem óleo, sendo utilizadas como branco na análise. $\mathrm{O}$ cálculo do rendimento de recuperação $(\mathrm{R}(\%))$ foi realizado com o uso da Equação 1, onde $\mathrm{C}_{\text {real }}$ representa a concentração de óleo de café torrado determinada na análise por $\mathrm{Uv}-\mathrm{V}$ is $(\mathrm{mg} / \mathrm{mL})$ e $\mathrm{C}_{\text {teórica }}$ representa a concentração de óleo de café torrado inicialmente adicionado à formulação $(\mathrm{mg} / \mathrm{mL})$. A análise foi realizada em duplicata.

$$
R(\%)=\frac{C_{\text {real }}}{C_{\text {teórica }}} \times 100
$$

\subsection{Figuras de mérito}

Especificidade: espectros dos componentes da formulação das nanocápsulas foram obtidos por varredura de 600 a $200 \mathrm{~nm}$ em um espectrofotômetro. Foi determinado o comprimento de onda onde o óleo de café torrado apresentasse maior absorbância sem sobreposição dos demais componentes da formulação. Linearidade: foram preparadas soluções, com concentrações de 0,$500 ; 0,300 ; 0,170 ; 0,100 ; 0,040$ e $0,020 \mathrm{mg} / \mathrm{mL}$, de óleo de café torrado em uma mistura de diclorometano e metanol (1:1) em triplicata. As soluções foram avaliadas via espectrofotometria UV-Vis (absorbância determinada na análise de especificidade). Limites de detecção (LD) de quantificação (LQ): foram determinados pelas Equações 2 e 3, onde: b, representa o coeficiente angular da curva de calibração e $S$, a variância residual calculada a partir do desvio padrão da leitura de amostras em branco (em média sete análises).

$$
\begin{aligned}
& L D=S \cdot \frac{3,3}{b} \\
& L Q=S \cdot \frac{10}{b}
\end{aligned}
$$

Precisão: A precisão foi estimada em dois níveis: 1) Precisão intermediária: os desvios padrões relativos (DPR\%) de três soluções $(0,500 ; 0,100$ e $0,020 \mathrm{mg} / \mathrm{mL})$ foram determinados com o mesmo equipamento em dias diferentes (inter-dia); 2) Reprodutibilidade: a mesma metodologia foi realizada porém em laboratórios diferentes (intra-laboratório) (Universidade Tecnológica Federal do Paraná-Campus Campo Mourão-Laboratório de Análise Instrumental I e Universidade Federal de Santa Catarina-Laboratório de Controle de Processos). Exatidão: foram realizados ensaios em triplicata em três níveis de concentração conhecida $(0,500 ; 0,100$ e $0,020 \mathrm{mg} / \mathrm{mL})$. A exatidão (E(\%)) foi determinada com a Equação 4, onde $\mathrm{C}_{\exp }(\mathrm{mg} / \mathrm{mL})$ é a concentração experimentalmente obtida (calibração) e $\mathrm{C}_{\text {teórica }}(\mathrm{mg} / \mathrm{mL})$ é a concentração teórica correspondente.

$$
E(\%)=\frac{C_{\text {exp }}}{C_{\text {teórica }}} x 100
$$

\section{RESULTADOS}

Os espectros normalizados dos componentes puros da formulação de nanocápsulas estão apresentados na Figura 1. Comparando os espectros é possível concluir que o comprimento de onda de absorção máxima para análises de óleo de café, onde há menos 


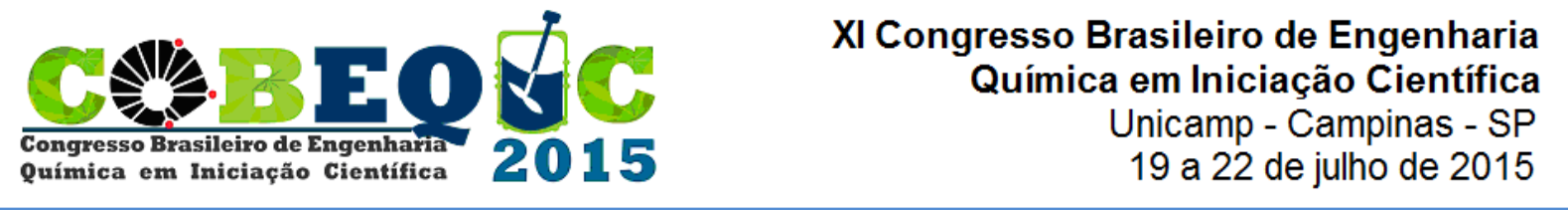

interferências dos demais componentes da formulação fica em $285 \mathrm{~nm}$. Sendo assim este comprimento de onda foi utilizado para determinar a concentração de óleo nas demais análises. As três curvas de calibração obtidas a $285 \mathrm{~nm}$ estão apresentadas na Figura 2 (a), juntamente com as respectivas equações de reta e coeficientes de correlação. Para avaliação estatística do ajuste linear das curvas, analisou-se o gráfico de valores preditos em função dos valores encontrados, considerando um intervalo de confiança de 95\% (Figura 2 (b)), bem como o gráfico dos resíduos do sinal analítico (Figura 2 (c)).

Figura 1. Espectros (UV-Vis): (a) óleo de café torrado $(0,4 \mathrm{mg} / \mathrm{mL})$, (b) lecitina de soja $(0,2 \mathrm{mg} / \mathrm{mL}),(\mathrm{c})$ PLLA $(0,4 \mathrm{mg} / \mathrm{mL})$

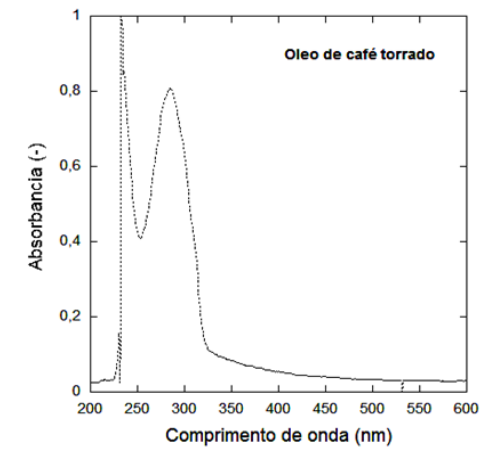

(a)

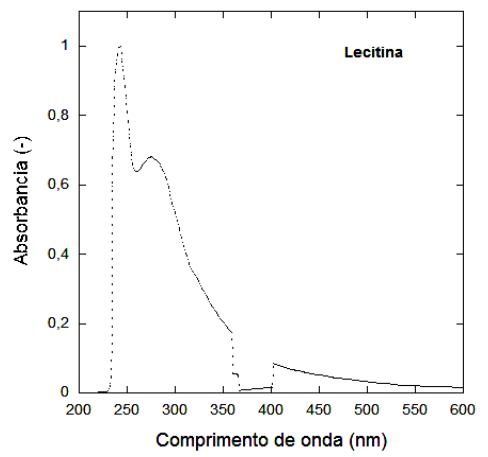

(b)

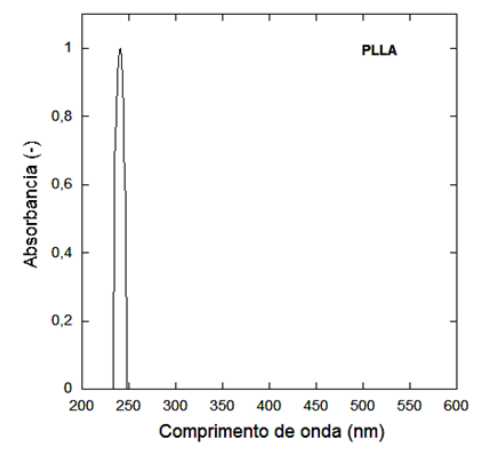

(c)

Figura 2 - (a) Curvas de calibração (triplicata); (b) Relação entre os valores observados e preditos (intervalo de confiança de 95\%); (c) Gráfico dos resíduos para os valores preditos

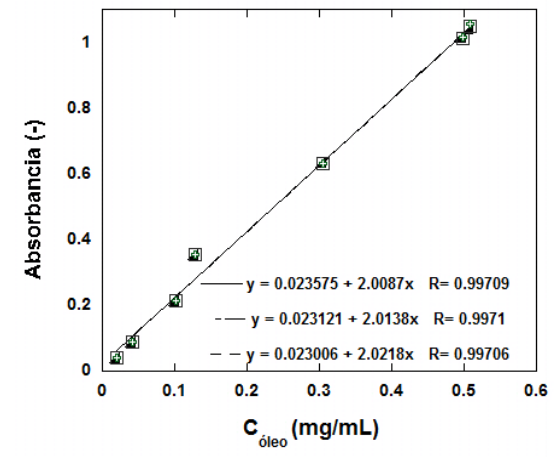

(a)

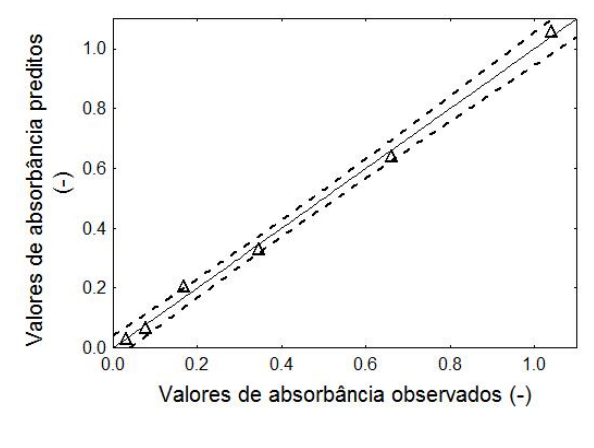

(b)

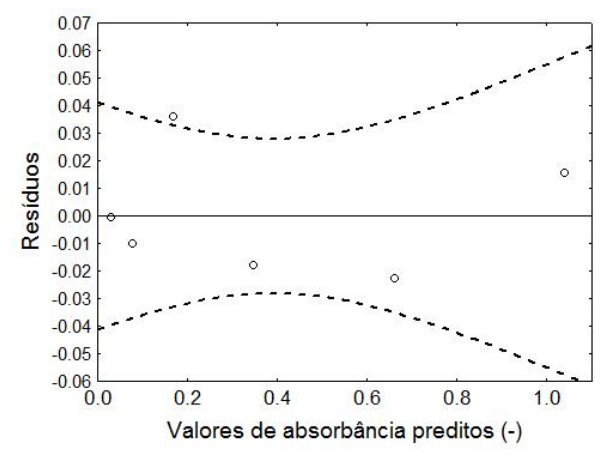

(c)

A análise de variância dos resultados obtidos na regressão linear apresentou $\mathrm{p}<4.10^{-5} \mathrm{a}$ um nível de confiança de $95 \%$. Este resultado demostra que o modelo linear é altamente significativo para descrição dos dados (Milagres et al., 2012). As três curvas de calibração obtidas apresentaram coeficientes de correlação dentro dos limites exigidos pela legislação específica e pelas agências reguladoras (Anvisa, 2003, Inmetro, 2003). Através da análise da Figura 2 (b) é possível concluir que há uma distribuição normal entre os valores previstos pelo modelo linear de ajuste para os dados obtidos em laboratório. Esta hipótese é confirmada pela avaliação da Figura 2 (c), obtida pelo delineamento do comportamento de distribuição dos resíduos, que representam a estimativa de erro e dos valores que não são explicados pelo modelo. Os valores de limite de quantificação (LQ) e limite de detecção (LD) encontrados foram, 0,0050 e $0,0016 \mathrm{mg} / \mathrm{mL}$, respectivamente. Sendo assim, o método permite analisar amostras com concentração de até $0,0016 \mathrm{mg} / \mathrm{mL}$, porém a quantificação é feita somente a partir de $0,0050 \mathrm{mg} / \mathrm{mL}$. Na Tabela 1 são apresentados os valores obtidos na avaliação da 
exatidão para validação do método proposto. Foram avaliadas três concentrações da curva de calibração nesta análise: mínima $(0,020 \mathrm{mg} / \mathrm{mL})$, intermediária $(0,100 \mathrm{mg} / \mathrm{mL})$ e máxima $(0,500 \mathrm{mg} / \mathrm{mL})$. As concentrações máxima e intermediária apresentaram valores de exatidão superiores a $98 \%$ de recuperação percentual. A concentração mínima, porém, apresentou baixa exatidão $(75,47 \%)$. A precisão intermediária e a reprodutibilidade têm seus dados apresentados na Tabela 2.

Tabela 1 - Avaliação da exatidão do método

\begin{tabular}{ccc}
\hline $\mathrm{C}_{\text {teórica }}(\mathrm{mg} / \mathrm{mL})$ & $\mathrm{C}_{\text {exp }}(\mathrm{mg} / \mathrm{mL})$ & $\mathrm{E}(\%)$ \\
\hline 0,500 & $0,492 \pm 0,008$ & 98,49 \\
0,100 & $0,099 \pm 0,041$ & 98,99 \\
0,020 & $0,015 \pm 0,062$ & 75,47 \\
\hline
\end{tabular}

Tabela 2 - Avaliação precisão intermediária (inter-dia) e reprodutibilidade (intra-laboratório)

\begin{tabular}{cccccc}
\hline & \multicolumn{4}{c}{ Laboratório 1 } & \multicolumn{3}{c}{ Laboratório 2 } & Reprodutibilidade \\
\cline { 2 - 5 } $\mathrm{C}_{\text {oléo }}(\mathrm{mg} / \mathrm{mL})$ & Dia 1 & Dia 2 & $\begin{array}{c}\text { Precisão } \\
\text { intermediária } \\
\text { (DPR\%) }\end{array}$ & Dia 1 & \\
\hline 0,500 & 1,308 & 0,715 & 1,011 & 1,002 & 1,007 \\
0,100 & 1,963 & 0,527 & 1,245 & 1,349 & 1,297 \\
0,020 & 2,830 & 3,928 & 3,379 & 12,60 & 7,99 \\
\hline
\end{tabular}

Todos os valores de DPR\% estão dentro da faixa de 5\%, estabelecido como aceitável para metodologias analíticas, pela ANVISA (Anvisa, 2003). Apenas o desvio padrão relativo para reprodutibilidade de análise da determinação do menor nível de concentração de óleo de café torrado $(0,020 \mathrm{mg} / \mathrm{mL})$, foi considerado como fora da faixa de aceitação. Como já foi verificado na avaliação da exatidão esta concentração apresentou os mais baixos valores de exatidão para o método, assim era esperado que as demais avaliações nesta concentração pudessem apresentar diferenças significativas. As nanocápsulas de óleo de café torrado produzidas foram caracterizadas quanto ao Dz sendo encontrado valor igual a $290 \pm 63 \mathrm{~nm}$. O IPD das mesmas foi de $0,34 \pm 0,16$, indicando uma ampla faixa de distribuição de tamanhos, o que de acordo com Leimann et al. (2013) ocorre em função da viscosidade da fase orgânica que afeta o quebramento das gotas da fase orgânica durante o processo de dispersão ou miniemulsificação. $\mathrm{O}$ rendimento de recuperação determinado foi de $104,5 \pm 2,1 \%$. $\mathrm{O}$ resultado mostra que não houveram perdas por volatilização do óleo durante o procedimento de encapsulação, ou seja, todo o óleo adicionado à formulação no início do procedimento de encapsulação (etapa de dispersão da fase orgânica na fase aquosa) permaneceu até o momento da análise.

\section{CONCLUSÕES}

Pode-se concluir que a metodologia analítica desenvolvida para quantificação do óleo de café torrado presente na formulação de nanocápsulas é adequado. Tendo em vista o resultado obtido para o rendimento de recuperação em conjunto com a validação do método também pode-se concluir que o método de miniemulsificação/evaporação do solvente é apropriado para encapsular óleo de café torrado.

\section{AGRADECIMENTOS}

Os autores agradecem à CAPEs, Fundação Araucária e CNPq/PIBIC. 


\section{REFERÊNCIAS}

BHUMIRATANA, N.; KOUSHIK A.; Chambers, E. Evolution of sensory aroma attributes from coffee beans to brewed coffee. LWT-Food Sci. and Technol. v. 44, p. 2185-2192, 2011.

ANVISA - Agência Nacional de Vigilância Sanitária, BRASIL. Resolução RE no 899 de 29/5/2003. Diário Oficial da União, Brasília, DF, 02/06/2003, seção 1 - Agência Nacional de Vigilância Sanitária (ANVISA) aprova o Guia para validação de métodos analíticos e bioanalíticos.

CHAIYASAT, P.; CHAIYASAT, A.; TEEKA, P.; NOPPALIT, S.; SRINORACHUN, U. Preparation of Poly(1-Lactic Acid) Microencapsulated Vitamin E. Energy Procedia. v. 34, p. 656-63, 2013.

CHORNY, M.; FISHBEIN, I.; DANENBERG, H. D.; GOLOMB, G. Lipophilic drug loaded nanospheres prepared by nanoprecipitation: effect of formulation variables on size, drug recovery and release kinetics. J. Control. Release, v. 83, p. 389-400, 2002.

CLARKE, R. J. Coffee: Recent Developments. Londres: Blackwell Science, 2001.

FARAH, A. Functional and Speciality beverage technology. USA: Woodhead Publishing Limited and CRC Press LLC, 2009.

DA SILVA-BUZANELLO, R. A.; FERRO, A. C.; BONA, E.; CARDOZO-FILHO, L.; DE ARAÚJO, P. H. H.; LEIMANN, F. V.; GONÇALVES, O. H. Validation of an Ultravioletvisible (UV-Vis) technique for the quantitative determination of curcumin in poly (1-lactic acid) nanoparticles. Food Chem. v. 172, p. 99-104, 2015.

INMETRO. Instituto Nacional de Metrologia, Normalização e Qualidade Industrial. Orientações sobre Validação de Métodos de Ensaios Químicos, DOQ-CGCRE-008, 2003.

LEIMANN, F. V.; CARDOZO FILHO, L.; SAYER, C.; ARAÚJO, P. H. H. Poly (3hydroxybutyrate-co-3-hydroxyvalerate) nanoparticles prepared by a miniemulsion/solvent evaporation technique: effect of phbv molar mass and concentration. Braz. J. Chem. Eng., v. 30, p. 369-377, 2013.

MADENE, A.; JACQUOT, M.; SCHER, J.; DESOBRY, S. Flavor encapsulation and controlled release - a review. Int. J. Food Sci. Tecnol., v. 41, p. 1-21, 2006.

MAHAPATRO, A.; SINGH, D. K. Biodegradable nanoparticles are excellent vehicle for site directed in-vivo delivery of drugs and vaccines. J Nanobiotechnology, v. 9, p. 1-11, 2011.

MALVERN INSTRUMENTS, 2011, at <http://www.biophysics.bioc.cam.ac.uk/wpcontent/uploads/2011/02/DLS_Terms_defined_Malvern.pdf $>$.

MilagreS, M. P.; BRANDÃO, S. C. C.; MAGAlhÃES, M. A.; MINIM, V. P. R.; MINIM, L. A. Development and validation of the high performance liquid chromatographyion exclusion method for detection of lactic acid in milk. Food chem., v. 135(3), 1078-1082, 2012.

TAWAKKAL, I. S.M.; CRAN, M.J.; MILTZ, J.; BIGGER, S. W. A review of poly(lactic acid)-based materials for antimicrobial packaging. J Food Sci., v. 79, p. 1477-90, 2014.

VALDERRAMA, P.; BRAGA, J. W. B.; POPPI, R. J. Estado da arte de figuras de mérito em calibração multivariada. Quim. Nova, v. 32, p. 1278-1287, 2009. 